\title{
CHARACTERIZATION OF THE FOURIER SERIES OF A DISTRIBUTION HAVING A VALUE AT A POINT
}

\author{
RICARDO ESTRADA
}

(Communicated by J. Marshall Ash)

\begin{abstract}
Let $f$ be a periodic distribution of period $2 \pi$. Let $\sum_{n=-\infty}^{\infty} a_{n} e^{i n \theta}$ be its Fourier series. We show that the distributional point value $f\left(\theta_{0}\right)$ exists and equals $\gamma$ if and only if the partial sums $\sum_{-x \leq n \leq a x} a_{n} e^{i n \theta_{0}}$ converge to $\gamma$ in the Cesàro sense as $x \rightarrow \infty$ for each $a>0$.
\end{abstract}

\section{INTRODUCTION}

The study of the relationship between the local behavior of a periodic function at a point and the convergence and summability of the corresponding Fourier series has been one of the main concerns of classical harmonic analysis, a subject with many years of history.

In particular, the characterization of the Fourier series having a "value" at a point is a very interesting problem, whose solution depends on the notion of value used. Among the various useful notions of value we could mention the value of a continuous function at a point, the measure-theoretical approximate value of an integrable function, or the distributional point value of a generalized function.

In this article we shall be concerned with the characterization of the Fourier series of generalized functions having a distributional point value. The notion of distributional point value was introduced by Łojasiewicz [8] and corresponds, roughly, to the existence of value "on the average". If $f \in \mathcal{D}^{\prime}(\mathbb{R})$ is a distribution and $x_{0} \in \mathbb{R}$, we say that $f$ has the distributional value $\gamma$ at $x=x_{0}$, and write $f\left(x_{0}\right)=\gamma$ in $\mathcal{D}^{\prime}$ if for each $\phi \in \mathcal{D}$ we have $\lim _{\varepsilon \rightarrow 0}\left\langle f\left(x_{0}+\varepsilon x\right), \phi(x)\right\rangle=\gamma \int_{-\infty}^{\infty} \phi(x) d x$ (if $f$ is given by an integral and $\int_{-\infty}^{\infty} \phi(x) d x=1$ this means that the "average" $\int_{-\infty}^{\infty} f\left(x_{0}+\varepsilon x\right) \phi(x) d x$ tends to $\gamma$ ). It can be shown that $f\left(x_{0}\right)=\gamma$ in $\mathcal{D}^{\prime}$ if and only if there exists a primitive of order $k$ of $f$, i.e., $F^{(k)}=f$, which is continuous in a neighborhood of $x=x_{0}$ and satisfies $\lim _{x \rightarrow x_{0}} k !\left(x-x_{0}\right)^{-k} F(x)=\gamma$. This corresponds to the notion of generalized derivatives (see [13] and the references therein) used in the theory of trigonometric series well before the definition of Łojasiewicz and even before the introduction of the notion of distributions by Schwartz [10].

It turns out that the existence of distributional point values is equivalent to the existence in the Cesàro sense of the limits of certain partial sums of the corresponding Fourier series. Convergence in the Cesàro sense is also a kind of "convergence

Received by the editors May 2, 1994 and, in revised form, October 18, 1994

1991 Mathematics Subject Classification. Primary 46F10, 42A24. 
on the average" and is defined as follows [6]: if $f$ is an integrable function defined for $x \geq 0$ we say that $F(x)$ tends to $L$ in the $(C, 1)$ sense as $x \rightarrow+\infty$, and write $\lim _{x \rightarrow+\infty} F(x)=L(C, 1)$ if $\lim _{x \rightarrow+\infty} \frac{1}{x} \int_{0}^{x} F(t) d t=L$. Convergence in the $(C, k)$ sense, $k=2,3, \ldots$, is defined recursively by $\lim _{x \rightarrow \infty} F(x)=L(C, k)$ if $\lim _{x \rightarrow+\infty} \frac{1}{x} \int_{0}^{x} F(t) d t=L(C, k-1)$. We say that $F(x)$ converges to $L$ in the Cesàro sense and write $\lim _{x \rightarrow+\infty} F(x)=L(C)$ if $\lim _{x \rightarrow+\infty} F(x)=L(C, k)$ for some $k$.

The Cesàro convergence of a sequence $\left\{x_{n}\right\}$ can be defined similarly and it is equivalent to the Cesàro convergence of the function $F$ defined by $F(t)=x_{\llbracket t \rrbracket}$. In particular a series $\sum_{n=1}^{\infty} a_{n}$ is Cesàro summable to the sum $S$, written as $\sum_{n=1}^{\infty} a_{n}=$ $S(C)$, if $\lim _{n \rightarrow \infty} \sum_{j=1}^{n} a_{j}=S(C)$. A series $\sum_{n=-\infty}^{\infty} a_{n}$ is $(C)$ summable to $S$ if both series $\sum_{n=0}^{\infty} a_{n}$ and $\sum_{n=1}^{\infty} a_{-n}$ are $(C)$ summable to $S_{1}$ and $S_{2}$, respectively, with $S=S_{1}+S_{2}$. A series $\sum_{n=-\infty}^{\infty} a_{n}$ is principal value Cesàro summable to $S$, written as p.v. $\sum_{n=-\infty}^{\infty} a_{n}=S(C)$, if $\lim _{n \rightarrow \infty} \sum_{j=-n}^{n} a_{j}=S(C)$.

Now let $f \in \mathcal{S}^{\prime}$ be a periodic distribution of period $2 \pi$ and let $\sum_{n=-\infty}^{\infty} a_{n} e^{i n \theta}$ be the associated Fourier series. Let $\theta_{0} \in \mathbb{R}$. Then the following two results are known $[3,12]$ :

(1) If $\sum_{n=-\infty}^{\infty} a_{n} e^{i n \theta_{0}}=\gamma(C)$, i.e., if the partial sums $\sum_{n=-N}^{M} a_{n} e^{i n \theta_{0}}$ tend to $\gamma$ in the Cesàro sense as $N$ and $M$ tend to infinity independently, then $f\left(\theta_{0}\right)=\gamma$ in $\mathcal{D}^{\prime}$. The converse does not hold $\left(f(\theta)=\sum_{n=2}^{\infty} \frac{e^{i n \theta}}{n \log n}\right.$, at $\theta=0$, is an example).

(2) If $f\left(\theta_{0}\right)=\gamma$ in $\mathcal{D}^{\prime}$, then p. v. $\sum_{n=-\infty}^{\infty} a_{n} e^{i n \theta_{0}}=\gamma(C)$, i.e.,

$$
\lim _{N \rightarrow \infty} \sum_{n=-N}^{N} a_{n} e^{i n \theta_{0}}=\gamma(C) .
$$

The converse does not hold $\left(f(\theta)=\sum_{n=1}^{\infty} n \sin n \theta\right.$, at $\theta=0$, is an example).

Here we prove the following

Theorem. Let $f \in \mathcal{S}^{\prime}$ be a periodic distribution of period $2 \pi$ and let $\sum_{n=-\infty}^{\infty} a_{n} e^{i n \theta}$ be its Fourier series. Let $\theta_{0} \in \mathbb{R}$. Then

$$
f\left(\theta_{0}\right)=\gamma, \quad \text { in } \mathcal{D}^{\prime}
$$

if and only if there exists $k$ such that

$$
\lim _{x \rightarrow+\infty} \sum_{-x \leq n \leq a x} a_{n} e^{i n \theta_{0}}=\gamma, \quad(C, k)
$$

for each $a>0$.

Our approach is based on the theory of distributional asymptotic expansions $[4,5,11]$ and is inspired by the work of Ramanujan [9] who was one of the first to study a sequence $\left\{a_{n}\right\}$ by studying the asymptotic behavior of series of the type $\sum_{n=1}^{\infty} a_{n} \phi(n \varepsilon)$, as $\varepsilon \rightarrow 0^{+}$, for smooth $\phi$. See also $[1,2]$. Here we study the Fourier series $\sum_{n=-\infty}^{\infty} a_{n} e^{i n \theta_{0}}$ by analysing the behavior as $\varepsilon \rightarrow 0$ of the series $\sum_{n=-\infty}^{\infty} a_{n} e^{i n \theta_{0}} \phi(n \varepsilon)$ for $\phi \in \mathcal{S}$.

Notice that we use the standard notation concerning spaces of distributions and test functions $[7,10]$. 


\section{Asymptotically homogeneOUs FunCtions}

In this section we define and give the basic properties of the asymptotically homogeneous functions of degree 0 . These functions play a central role in our analysis.

Lemma 1. Let $\tau$ be a real-valued continuous function defined in an interval of the form $[A, \infty)$ for some $A \in \mathbb{R}$. Suppose

$$
\tau(a x)=a^{\mu} \tau(x)+o(1), \quad \text { as } x \rightarrow+\infty,
$$

for each $a>0$. If $\mu<0$, then

$$
\tau(x)=o(1), \quad \text { as } x \rightarrow+\infty .
$$

Proof. Let $\varepsilon>0$. Let $x_{0}>0$ be such that $\left|\tau(2 x)-2^{\mu} \tau(x)\right| \leq \varepsilon$ for $x>x_{0}$. Let $M=\max \left\{|\tau(x)|: x_{0} \leq x \leq 2 x_{0}\right\}$. An inductive argument shows that if $x \in\left[2^{n} x_{0}, 2^{n+1} x_{0}\right], n=0,1,2, \ldots$, then $|\tau(x)| \leq 2^{n \mu} M+\sum_{j=1}^{n-1} 2^{j \mu} \varepsilon$. Therefore, $\lim _{x \rightarrow+\infty}|\tau(x)| \leq \frac{2^{\mu}}{1-2^{\mu}} \varepsilon$ and (2.2) follows.

The lemma does not hold if $\mu=0$. Indeed, there are functions like $\ln (\ln x)$, $|\ln x|^{\alpha}, \alpha<1$, or $\cos \sqrt{|\ln x|}$ that satisfy $\tau(a x)=\tau(x)+o(1)$, as $x \rightarrow+\infty$, for each $a>0$, but which do not tend to zero at infinity.

Definition. Let $\tau$ be a continuous function defined in an interval of the form $[A, \infty)$ for some $A \in \mathbb{R}$. We say that $\tau$ is asymptotically homogeneous of degree 0 if for each $a>0$ we have

$$
\tau(a x)=\tau(x)+o(1), \quad \text { as } x \rightarrow+\infty .
$$

The asymptotically homogeneous functions of degree 0 are related to the slowly oscillating functions [5], also known as regularly varying functions of order 0 . These are positive functions that satisfy $\rho(a x)=\rho(x)+o(\rho(x))$, as $x \rightarrow \infty$, for each $a>0$. However, the two concepts are different: $\ln x$ is slowly oscillating but not asymptotically homogeneous of degree 0 while $\cos \sqrt{|\ln x|}$ is asymptotically homogeneous of degree 0 but not slowly oscillating.

Observe that the argument of the proof of Lemma 1 shows that if $\tau$ is an asymptotically homogeneous function of order 0 , then $\tau(x)=o(\ln x)$, as $x \rightarrow+\infty$.

Notice also that we did not ask for any uniform behavior with respect to $a$ in (2.3). The fact that (2.3) holds uniformly on $a \in[A, B]$ if $[A, B] \subseteq(0, \infty)$ follows from the definition, as we shall see.

Lemma 2. Let $\tau$ be an asymptotically homogeneous function of degree 0, continuous in $[0, \infty)$. Let $H$ be the Heaviside function. Then

$$
\tau(\lambda x) H(x)=\tau(\lambda) H(x)+o(1), \quad \text { as } \lambda \rightarrow \infty, \text { in } \mathcal{S}^{\prime} .
$$

If $[A, B] \subseteq(0, \infty)$, then (2.3) holds uniformly for $a \in[A, B]$.

Proof. Suppose first that $\tau$ is bounded in $[0, \infty)$. Then if $\phi \in \mathcal{S}^{\prime}$ we can apply the Lebesgue dominated convergence theorem to obtain

$$
\lim _{\lambda \rightarrow \infty} \int_{0}^{\infty}(\tau(\lambda x)-\tau(\lambda)) \phi(x) d x=0 .
$$

This is $(2.4)$

The uniform convergence follows from the distributional formula (2.4). Indeed, weak convergence in $\mathcal{S}^{\prime}$ implies strong convergence [10]. Thus if $K$ is a 
compact subset of $\mathcal{S}$, then $\langle\tau(\lambda x) H(x)-\tau(\lambda) H(x), \phi(x)\rangle=o(1)$ uniformly for $\phi \in K$. Let $\phi_{0} \in \mathcal{S}$ be a function that satisfies $\int_{0}^{\infty} \phi_{0}(x) d x=1$. Then if $[A, B] \subseteq(0, \infty)$ the set $K=\left\{a^{-1} \phi_{0}\left(a^{-1} x\right): a \in[A, B]\right\}$ is a compact set of $\mathcal{S}$. Then $\left\langle\tau(\lambda x) H(x), \phi_{0}(x)\right\rangle=\tau(\lambda)+o(1)$ as $\lambda \rightarrow \infty$, and so $\tau(\lambda a)=\left\langle\tau(\lambda a x), \phi_{0}(x)\right\rangle+$ $o(1)=\left\langle\tau(\lambda x), a^{-1} \phi_{0}\left(a^{-1} x\right)\right\rangle+o(1)=\tau(\lambda)+o(1)$ uniformly for $a \in[A, B]$.

Let us now return to the general case when $\tau$ is not necessarily bounded. We shall first show that if $[A, B] \subseteq(0, \infty)$, then $\tau(a x)=\tau(x)+o(1)$, as $x \rightarrow+\infty$, uniformly on $a \in[A, B]$. Observe first that the functions $\cos \tau(x)$ and $\sin \tau(x)$ are bounded asymptotically homogeneous functions. By what we have already proven it follows that if $[A, B] \subseteq(0, \infty)$, then $e^{i \tau(a x)}=e^{i \tau(x)}+o(1)$, as $x \rightarrow \infty$, uniformly on $a \in[A, B]$. Let $\varepsilon>0$. Suppose $\varepsilon<\pi$ and $A<1<B$. There exists $x_{0}>0$ such that $\left|e^{i \tau(a x)}-e^{i \tau(x)}\right| \leq\left|1-e^{i \varepsilon}\right|$ for $x \geq x_{0}$ and $a \in[A, B]$. For each $x \geq x_{0}$ the set $\{\tau(a x): A \leq a \leq B\}$ is a connected set contained in $\bigcup_{n=-\infty}^{\infty}[\tau(x)-\varepsilon+2 n \pi, \tau(x)+\varepsilon+2 n \pi]$ and it follows that it is contained in the component that contains $\tau(x)$; that is, $|\tau(a x)-\tau(x)| \leq \varepsilon$, for $a \in[A, B]$.

To prove (2.4) it would be enough to prove that there are constants $A_{0}, A_{1}$ such that $|\tau(\lambda x)-\tau(\lambda)| \leq A_{0}|\ln x|+A_{1}$ for $x>0$ and $\lambda>\lambda_{0}$ for then the dominated convergence theorem could be invoked again.

Let $\lambda_{0}>0$ be such that $|\tau(\lambda x)-\tau(\lambda)| \leq 1$ if $\lambda \geq \lambda_{0}$ and $x \in[1 / 2,2]$. Then it follows by induction that $|\tau(\lambda x)-\tau(\lambda)| \leq n+1$ if $x \in\left[2^{n}, 2^{n+1}\right], \lambda \geq \lambda_{0}, n=$ $0,1,2 \ldots$ Therefore $|\tau(\lambda x)-\tau(\lambda)| \leq \frac{|\ln x|}{\ln 2}+1$ if $x \geq 1, \lambda \geq \lambda_{0}$. Proceeding similarly, if $x \in\left[\frac{1}{2^{n+1}}, \frac{1}{2^{n}}\right], \lambda \geq 2^{n} \lambda_{0}, n=0,1,2, \ldots$, then $|\tau(\lambda x)-\tau(\lambda)| \leq n+1 \leq \frac{|\ln x|}{\ln 2}+1$. Recall now that $\tau(x)=o(\ln x)$, as $x \rightarrow \infty$. Then we can find constants $M_{0}, M_{1}$ such that $|\tau(x)| \leq M_{0}|\ln x|+M_{1}, x>0$. Therefore, if $x \in\left[\frac{1}{2^{n+1}}, \frac{1}{2^{n}}\right]$ but $\lambda<2^{n} \lambda_{0}$, then $\lambda<\lambda_{0} / x$ and so $|\tau(\lambda x)-\tau(\lambda)| \leq 2\left(M_{0}|\ln \lambda|+M_{1}\right) \leq 2\left(M_{0}|\ln x|+M_{0}\left|\ln \lambda_{0}\right|+M_{1}\right)$. Summarizing, if $A_{0}=\max \left\{2 M_{0}, 1 / \ln 2\right\}, A_{1}=\max \left\{2\left(M_{0}\left|\ln \lambda_{0}\right|+M_{1}\right), 1\right\}$, then $|\tau(\lambda x)-\tau(\lambda)| \leq A_{0}|\ln x|+A_{1}$ for $x>0$ and $\lambda \geq \lambda_{0}$.

The asymptotically homogeneous functions of degree 0 do not have to be smooth, i.e., $C^{\infty}$. However, we have

Lemma 3. Let $\tau$ be an asymptotically homogeneous function of degree 0, continuous in $[0, \infty)$. Then there exists a function $\sigma$, asymptotically homogeneous of degree 0 , smooth in $[0, \infty)$, such that

$$
\tau(x)=\sigma(x)+o(1), \quad \text { as } x \rightarrow \infty .
$$

Proof. Define $\sigma(\lambda)=\int_{0}^{\infty} \tau(\lambda x) \phi_{0}(x) d x, \lambda \geq 1$, where $\phi_{0} \in \mathcal{S}$ satisfies $\int_{0}^{\infty} \phi_{0}(x) d x$ $=1$, and extend to $[0,+\infty)$ in any smooth way. Then (2.4) gives (2.5).

\section{Primitives of A Distributionally null Sequence}

If $\left\{f_{n}\right\}$ is a sequence of continuous functions that converge uniformly to zero on an interval of the form $[-A, A]$, then the sequence of primitives $\left\{\int_{0}^{x} f_{n}(t) d t\right\}$ also converges uniformly to zero while, in general, the sequence of derivatives $\left\{f_{n}^{\prime}\right\}$, even if defined, does not. On the other hand, the situation with the distributional convergence is the opposite: if $f_{n} \rightarrow 0$ in $\mathcal{S}^{\prime}$, then also $f_{n}^{\prime} \rightarrow 0$ in $\mathcal{S}^{\prime}$ but the sequence of primitives $\left\{\int_{0}^{x} f_{n}(t) d t\right\}$, even if defined, might be divergent. We now turn our attention to the study of this last problem.

Let $F \in \mathcal{S}^{\prime}$. If $F$ is integrable near $x=0$, then we can define the primitive $\int_{0}^{x} F(t) d t$. If $F$ is more singular near $x=0$, then there is no canonical way to 
define $\int_{0}^{x} F(t) d t$, that is, there is no canonical way to single out a primitive of $F$ that vanishes at $x=0$. However, if $F$ is locally even near $x=0$, it has a unique locally odd primitive, which we denote as $\int_{0}^{x} F(t) d t$. Observe that with this notation $\int_{0}^{x} \delta(t) d t=\frac{1}{2} \operatorname{sgn} x$, where $\operatorname{sgn} x=x /|x|, x \neq 0$, is the sign function. We shall use the notation $\int_{0}^{x} F(t) d t$ so long as $F=F_{0}+F_{1}$, where $F_{0}$ is locally integrable near $x=0$ and where $F_{1}$ is locally even.

Let $\left\{F_{n}\right\}_{n=1}^{\infty}$ be a sequence of distributions of $\mathcal{S}^{\prime}$. Suppose that $\lim _{n \rightarrow \infty} F_{n}=0$ in $\mathcal{S}^{\prime}$. Then [10] there are primitives of the $F_{n}$ that also tend to zero. In particular, if $\int_{0}^{x} F_{n}(t) d t$ is defined for each $n$, there are constants $c_{n}$ such that $\int_{0}^{x} F_{n}(t) d t=c_{n}+$ $o(1)$, as $n \rightarrow \infty$ in $\mathcal{S}^{\prime}$. The example $F_{n}(x)=-2 n x e^{-x^{2}}\left(1-e^{-x^{2}}\right)^{n-1}, \int_{0}^{x} F_{n}(t) d t=$ $1-\left(1-e^{-x^{2}}\right)^{n}=1+o(1)$ shows that the $c_{n}$ 's cannot be replaced by 0 , in general. Observe also that if the $F_{\lambda}$ depend smoothly on the parameter $\lambda$ and $F_{\lambda}=o(1)$, as $\lambda \rightarrow \infty$, then $\int_{0}^{x} F_{\lambda}(t) d t=\sigma(\lambda)+o(1)$, where $\sigma$ is smooth.

Lemma 4. Let $F_{0} \in \mathcal{S}^{\prime}$ be a Radon measure such that $\int_{0}^{x} F_{0}(t) d t$ is defined. Define the distributions $F_{n}, n \geq 1$, recursively by $F_{n}(x)=\int_{0}^{x} F_{n-1}(t) d t, n \geq 1$. Suppose

$$
F_{0}(\lambda x)=o(1 / \lambda), \quad \text { as } \lambda \rightarrow+\infty, \text { in } \mathcal{S}^{\prime} .
$$

Then there exists an asymptotically homogeneous function of degree $0, \sigma(\lambda)$, such that

$$
F_{n}(\lambda x)=\frac{\lambda^{n-1} x^{n-1} \sigma(\lambda)}{(n-1) !}+o\left(\lambda^{n-1}\right), \quad \text { as } \lambda \rightarrow+\infty, \text { in } \mathcal{S}^{\prime}
$$

for $n \geq 1$. There exists $n_{0}$ such that the convergence in $(3.2)$ is uniform on compacts for $n \geq n_{0}$. Conversely, if (3.2) holds for some $n \geq 1$, then $F_{0}(\lambda x)=o(1 / \lambda)$ in $\mathcal{S}^{\prime}$.

Proof. Suppose $F_{0}(\lambda x)=o(1 / \lambda)$ in $\mathcal{S}^{\prime}$. Then there exists a smooth function $\sigma(\lambda)$ such that $F_{1}(\lambda x)=\sigma(\lambda)+o(1)$, as $\lambda \rightarrow \infty$, in $\mathcal{S}^{\prime}$. Replacing $\lambda x$ by $\lambda x a$ and grouping in two different ways, we obtain $\sigma(a \lambda)=\sigma(\lambda)+o(1)$, as $\lambda \rightarrow \infty$ for each $a>0$. Thus $\sigma$ is asymptotically homogeneous of degree 0 . Hence (3.2) holds for $n=1$. Suppose now it holds for some $n \geq 1$. Then integrating again we obtain $F_{n+1}(\lambda x)=\lambda^{n} x^{n} \sigma(\lambda) / n !+\rho(\lambda)+o\left(\lambda^{n}\right)$, as $\lambda \rightarrow \infty$, for some function $\rho$. Evaluating at $\lambda a x$ thus yields $\rho(\lambda a)=\rho(\lambda)+o\left(\lambda^{n}\right)$ and thus by Lemma 1, applied to $\lambda^{-n} \rho(\lambda)$, it follows that $\rho(\lambda)=o\left(\lambda^{n}\right)$ and $(3.2)$ is obtained for $n+1$.

That the convergence in (3.2) is uniform for $x$ in compacts if $n$ is large follows by the definition of the convergence of distributions.

The converse is obtained by differentiating (3.2) $n$ times with respect to $x$.

Observe that if $F_{0}$ is even, then $F_{1}$ is odd and thus $\sigma(\lambda)=o(1)$, as $\lambda \rightarrow \infty$, and (3.2) becomes $F_{n}(\lambda x)=o\left(\lambda^{n-1}\right)$, as $\lambda \rightarrow \infty$. The same conclusion is obtained if $\operatorname{supp} F_{0} \subseteq[0, \infty)$.

The sequence of functions $\left\{F_{n}\right\}_{n=0}^{\infty}$ is also related to Cesàro summability. The following lemma is an immediate corollary of the results proved in [6, p. 110].

Lemma 5. Let $F_{0}$ be a function defined for $x>0$ that, suitably extended to $\mathbb{R}$, defines an element of $\mathcal{S}^{\prime}$ for which $\int_{0}^{x} F_{0}(t) d t$ is defined. Then $\lim _{x \rightarrow+\infty} F_{0}(x)=\gamma$ $(C, n)$ if and only if $\lim _{x \rightarrow \infty} n ! x^{-n} F_{n}(x)=\gamma$.

\section{THe MAIN RESUlT}

In this section we apply the results of the previous sections to characterize the Fourier series of the periodic distributions having a distributional point value. 
Lemma 6. Let $f \in \mathcal{S}^{\prime}$ be a periodic distribution of period $2 \pi$ and let $\sum_{n=-\infty}^{\infty} a_{n} e^{i n \theta}$ be its Fourier series. Let $\theta_{0} \in \mathbb{R}$. Then

$$
f\left(\theta_{0}\right)=\gamma, \quad \text { in } \mathcal{D}^{\prime}
$$

if and only if

$$
\sum_{n=-\infty}^{\infty} a_{n} e^{i n \theta_{0}} \delta(\lambda x-n)=\frac{\gamma \delta(x)}{\lambda}+o(1 / \lambda), \quad \text { as } \lambda \rightarrow \infty, \text { in } \mathcal{S}^{\prime} .
$$

Proof.

$$
\begin{aligned}
f\left(\theta_{0}\right) & =\gamma, \quad \text { in } \mathcal{D}^{\prime} \\
& \Leftrightarrow \lim _{\varepsilon \rightarrow 0}\left\langle f\left(\theta_{0}+\varepsilon \theta\right), \phi(\theta)\right\rangle=\langle\gamma, \phi(\theta)\rangle \quad \forall \phi \in \mathcal{D} \\
& \Leftrightarrow \lim _{\varepsilon \rightarrow 0}\left\langle f\left(\theta_{0}+\varepsilon \theta\right), \phi(\theta)\right\rangle=\langle\gamma, \phi(\theta)\rangle \quad \forall \phi \in \mathcal{S} \\
& \Leftrightarrow \lim _{\varepsilon \rightarrow 0} \sum_{n=-\infty}^{\infty} a_{n} e^{i n \theta_{0}}\left\langle e^{i n \varepsilon \theta}, \phi(\theta)\right\rangle=\gamma \int_{-\infty}^{\infty} \phi(x) d x \quad \forall \phi \in \mathcal{S} \\
& \Leftrightarrow \lim _{\varepsilon \rightarrow 0} \sum_{n=-\infty}^{\infty} a_{n} e^{i n \theta_{0}} \hat{\phi}(n \varepsilon)=\gamma \hat{\phi}(0) \quad \forall \phi \in \mathcal{S} \\
& \Leftrightarrow \lim _{\varepsilon \rightarrow 0} \sum_{n=-\infty}^{\infty} a_{n} e^{i n \theta_{0}} \psi(n \varepsilon)=\gamma \psi(0) \quad \forall \psi \in \mathcal{S} \\
& \Leftrightarrow \lim _{\varepsilon \rightarrow 0} \sum_{n=-\infty}^{\infty} a_{n} e^{i n \theta_{0}} \delta(x-n \varepsilon)=\gamma \delta(x) \quad \text { in } \mathcal{S}^{\prime} \\
& \Leftrightarrow \sum_{n=-\infty}^{\infty} a_{n} e^{i n \theta_{0}} \delta(\lambda x-n)=\frac{\gamma \delta(x)}{\lambda}+o(1 / \lambda), \quad \text { as } \lambda \rightarrow \infty \text { in } \mathcal{S}^{\prime} .
\end{aligned}
$$

We are now ready to give our main result.

Theorem. Let $f \in \mathcal{S}^{\prime}$ be a periodic distribution of period $2 \pi$ and let $\sum_{n=-\infty}^{\infty} a_{n} e^{i n \theta}$ be its Fourier series. Let $\theta_{0} \in \mathbb{R}$. Then

$$
f\left(\theta_{0}\right)=\gamma, \quad \text { in } \mathcal{D}^{\prime},
$$

if and only if there exists $k$ such that

$$
\lim _{x \rightarrow \infty} \sum_{-x \leq n \leq a x} a_{n} e^{i n \theta_{0}}=\gamma \quad(C, k)
$$

for each $a>0$.

Proof. Let $F_{0}(x)=\sum_{n=-\infty}^{\infty} a_{n} e^{i n \theta_{0}} \delta(x-n)$ and define $F_{1}, F_{2}, \ldots$ recursively by $F_{n+1}(x)=\int_{0}^{x} F_{n}(t) d t$. Let $a>0$, put $G_{0}(x)=G_{0}(a, x)=\sum_{-x \leq n \leq a x} a_{n} e^{i n \theta_{0}}$ and define $G_{1}, G_{2}, \ldots$ recursively by $G_{n+1}(x)=\int_{0}^{x} G_{n}(t) d t$. Observe that (4.4) means that $\lim _{x \rightarrow \infty} k ! x^{-k} G_{k}(x)=\gamma$.

Suppose first that $f\left(\theta_{0}\right)=\gamma$. Then $F_{0}(\lambda x)=\gamma \delta(\lambda x)+o(1 / \lambda)$, as $\lambda \rightarrow \infty$, in $\mathcal{S}^{\prime}$. Using Lemma 4 , there exists an asymptotically homogeneous function of degree 0 such that if $n \geq 1$, then

$$
F_{n}(\lambda x)=\frac{\gamma \operatorname{sgn}(\lambda x)(\lambda x)^{n-1}}{2(n-1) !}+\frac{\sigma(\lambda)(\lambda x)^{n-1}}{(n-1) !}+o\left(\lambda^{n-1}\right), \quad \text { as } \lambda \rightarrow \infty, \text { in } \mathcal{S}^{\prime}
$$


and there exists $n_{0}$ such that if $n \geq n_{0}$ this holds uniformly on $x \in[-A, A]$ for $A>0$. Thus, if $n \geq n_{0}$,

$$
\begin{aligned}
G_{n-1}(x) & =a^{1-n} F_{n}(a x)-(-1)^{1-n} F_{n}(-x) \\
& =\frac{\gamma x^{n-1}}{2(n-1) !}+\frac{\sigma(x)}{(n-1) !}+\frac{\gamma}{2(n-1) !}-\frac{\sigma(x)}{(n-1) !}+o\left(x^{n-1}\right) \\
& =\frac{\gamma x^{n-1}}{(n-1) !}+o\left(x^{n-1}\right)
\end{aligned}
$$

and (4.4) follows with $k=n-1$.

Conversely, suppose (4.4) holds. Let $n=k+1$, so that

$$
\lim _{x \rightarrow \infty}(n-1) ! x^{1-n} G_{n-1}(x)=\gamma .
$$

Define $\tau(x)=(n-1) ! x^{1-n} F_{n}(x)-\gamma / 2$. Then $\tau$ is asymptotically homogeneous of degree 0 . Thus there exists a smooth asymptotically homogeneous function of degree 0 such that $\tau(x)=\sigma(x)+o(1)$, as $x \rightarrow \infty$. It follows that

$$
F_{n}(x)=\frac{\gamma \operatorname{sgn} x x^{n-1}}{2(n-1) !}+\frac{\sigma(x) x^{n-1}}{(n-1) !}+o\left(x^{n-1}\right), \quad \text { as }|x| \rightarrow \infty,
$$

and therefore, by Lemma 2,

$$
F_{n}(\lambda x)=\frac{\gamma \operatorname{sgn}(\lambda x)(\lambda x)^{n-1}}{2(n-1) !}+\frac{\sigma(\lambda)(\lambda x)^{n-1}}{(n-1) !}+o\left(\lambda^{n-1}\right), \quad \text { as } \lambda \rightarrow \infty, \text { in } \mathcal{S}^{\prime} .
$$

Differentiating $n$ times we obtain $F_{0}(\lambda x)=\gamma \delta(\lambda x)+o(1 / \lambda)$, as $\lambda \rightarrow \infty$, as required.

It is interesting to observe that (4.4) holds uniformly on $a \in[A, B]$ if $[A, B] \subseteq$ $(0, \infty)$ but that it is not necessary to assume such uniform behavior to obtain (4.3).

Notice also that the theorem remains valid if (4.4) holds uniformly for $a \in H$ where $H$ is a set dense in some interval, $\bar{H}=[A, B], 0<A<B<\infty$. In particular, $f\left(\theta_{0}\right)=\gamma$ if and only if

$$
\lim _{N \rightarrow \infty} \sum_{n=-\llbracket N / p \rrbracket}^{\llbracket N / q \rrbracket} a_{n} e^{i n \theta_{0}}=\gamma \quad(C, k)
$$

for each $p, q \in\{1,2,3, \ldots\}$, uniformly if $A<p / q<B$ for $[A, B] \subseteq(0, \infty)$. Observe that (4.5) considers the convergence, in the Cesàro sense, of sequences.

The following consequences are worth recording.

Corollary 1. If $f$ is symmetric about $\theta=\theta_{0}$, i.e., $f\left(\theta-\theta_{0}\right)=f\left(\theta_{0}-\theta\right)$, then $f\left(\theta_{0}\right)=\gamma$ in $\mathcal{D}^{\prime}$ if and only if $\sum_{n=-\infty}^{\infty} a_{n} e^{i n \theta_{0}}=\gamma(C)$.

Corollary 2. If $f$ is antisymmetric about $\theta=\theta_{0}$, i.e., $f\left(\theta-\theta_{0}\right)=-f\left(\theta_{0}-\theta\right)$, then $f\left(\theta_{0}\right)=\gamma$ in $\mathcal{D}^{\prime}$ if and only if there is a function $\sigma$, asymptotically homogeneous of degree 0 , such that $\sum_{n=1}^{N} a_{n} e^{i n \theta_{0}}=\sigma(N)+o(1)(C)$, as $N \rightarrow \infty$. In this case $\gamma=0$.

Corollary 3. If the Fourier series of $f$ is of the power series type, i.e., $f(\theta)=$ $\sum_{n=0}^{\infty} a_{n} e^{i n \theta}$, then $f\left(\theta_{0}\right)=\gamma$ in $\mathcal{D}^{\prime}$ if and only if $\sum_{n=0}^{\infty} a_{n} e^{i n \theta_{0}}=\gamma(C)$. 
RICARDO ESTRADA

\section{REFERENCES}

1. B. C. Berndt and R. J. Evans, Extensions of asymptotic expansions from Chapter 15 of Ramanujan's second notebook, J. Reine Angew. Math. 361 (1985), 118-134. MR 87b:41031

2. R. Estrada, The asymptotic expansion of certain series considered by Ramanujan, Appl. Anal. 43 (1992), 191-228. MR 95i:46053

3. _ Valores puntuales distribucionales de series de Fourier, Memorias II Encuentro Centroamericano de Matemáticos, San Ramón, Costa Rica, 1994.

4. R. Estrada and R. P. Kanwal, Asymptotic separation of variables, J. Math. Anal. Appl. 178 (1993), 130-142. MR 94g:46045

5. — Asymptotic analysi: A distributional approach, Birkhäuser, Boston, 1994. MR 95g: 46071

6. G. H. Hardy, Divergent series, Clarendon Press, Oxford, 1949. MR 11:25a

7. R. P. Kanwal, Generalized functions: Theory and technique, Academic Press, New York, 1983. MR 85f: 46001

8. S. Łojasiewicz, Sur la valeur et la limite d'une distribution en un point, Studia Math. 16 (1957), 1-36. MR 19:433d

9. S. Ramanujan, Notebooks, 2 vols., Bombay, 1957. MR 20:6340

10. L. Schwartz, Théorie des distributions Hermann, Paris, 1966. MR 35:730

11. V. S. Vladimirov, Y. N. Drozhinov, and B. I. Zavyalov Multidimensional Tauberian theorems for generalized functions, Nauka, Moscow, 1986. MR 87m:46092

12. G. Walter, Pointwise convergence of distributions, Studia Math. 26 (1966), 143-154. MR 32: 8036

13. A. Zygmund, Trigonometric series, 2 vols., Cambridge Univ. Press, Cambridge and New York, 1959. MR 21:6498

Department of Mathematics, Texas A \& M University, College Station, Texas 77843 Current address: P. O. Box 276, Tres Ríos, Costa Rica

E-mail address: restrada@cariari.ucr.ac.cr 